\title{
Research on Robust Optimization of Wind Farm Safety Operation Cost
}

\author{
Lu Yao, 2, a , Yang Taihua ${ }^{1, b}$ \\ ${ }^{1}$ Shanghai University of Electric Power, Shanghai \\ ${ }^{2}$ Shanghai Songjiang Power Supply Company of State Grid, Shanghai \\ a,b463501137@qq.com
}

Keywords: robust optimization; wind farm; operation security

\begin{abstract}
Robust optimization is an optimization method that uses interval disturbance information to make optimal decisions under worst-case disturbance conditions. As its advantages of easy access to basic data, high computational efficiency, and suitability for large-scale system solving, it has been applied for power system scheduling problems recently.
\end{abstract}

\section{Overview of Robust Optimization}

Generally speaking, the robust optimization model can be solved by transforming it into its deterministic equivalent model, and the solution scale is relatively small compared with the stochastic programming method. In order to avoid loss of generality, first, take the linear programming problem with parametric uncertainties as an example to construct the signal model of robust optimization, which is expressed as:

$$
\left\{\left\{\mathrm{C}^{\mathrm{T}} \mathrm{X}: \mathrm{MX} \leq \mathrm{n}\right\}:(\mathrm{C}, \mathrm{M}, \mathrm{n}) \in \mathrm{U}\right\}
$$

In equation (1), $\mathrm{X}$ is $\mathrm{n}$ order pending decision vector; $\mathrm{C}$ is the parameter vector in the linear objective function. $\mathrm{M}$ is $\mathrm{m}$ by $\mathrm{n}$ order coefficient matrix of constraint equation. $\mathrm{N}$ is $\mathrm{m}$-order parameter vector; $U$ is the uncertainty set. As can be seen from equation (1), linear robust optimization model and linear programming model:

$$
\left\{\left\{\mathrm{C}^{\mathrm{T}} \mathrm{X}: \mathrm{MX} \leq \mathrm{n}\right\}\right.
$$

This model is consistent in form, but there are essential differences between the two parameter attributes. The robust optimization model considers the uncertainty of the parameters in the objective function and/or constraint conditions, that is, the parameters $\mathrm{C}, \mathrm{M}$ and $\mathrm{n}$ can be arbitrarily evaluated in the uncertain set $\mathrm{U}$. According to the definition of robust optimization, the solution has the following characteristics:

(1)A definite numerical solution can be obtained if the decision is made on the premise that the implementation of uncertain parameters is unknown;(2)Decision-making results are sufficient to cope with simultaneous disturbance of all uncertain parameters; (3) When the uncertainty parameter is evaluated in the predefined set of uncertainties, the constraint of the model must be satisfied. For robust optimization solution of the above characteristics, must explicit expression in robust optimization model parameter uncertainty to the worst effects of decision results, thus, the type (1) as shown in the robust optimization is transformed into the standard model of the robust peer model, as shown in the following type:

$$
\left\{\mathrm{C}^{\mathrm{T}} \mathrm{X}: \mathrm{MX} \leq \mathrm{n}\right\} \quad(\mathrm{C}, \mathrm{M}, \mathrm{n}) \in \mathrm{U}
$$

\section{Wind Power Robust Planning Model}

Wind speed data is often estimated by wind speed data measured in the short term. It has a certain randomness and contingency. If you entirely rely on this data to make decisions, it is very likely that the planned results are inconsistent with the actual situation, thereby you will not be able to obtain the most economic benefits. Therefore, when making optimization planning, it is 
necessary to consider the uncertainty of the wind speed to enhance the robustness of the planning results, that is, to adopt a robust optimization method for planning. The robust optimization model is as follows:

$$
\mathrm{C}^{\mathrm{T}} \mathrm{X}
$$

s.t

$$
\begin{gathered}
H(\mathrm{u}) \mathrm{x}=\mathrm{h}, \mathrm{u} \in \mathrm{U} \\
\mathrm{Gx} \leq \mathrm{g} \\
\leq \mathrm{x} \leq
\end{gathered}
$$

In the formula, $\mathrm{H}(\mathrm{u})$ is the coefficient matrix expressed as a function of $\mathrm{u}$. Objective function

$$
\mathrm{C}^{\mathrm{T}} \mathrm{X}
$$

is equivalent to

$$
\mathrm{C}^{\mathrm{T}} \mathrm{X}
$$

Thus, it can be transformed into a model:

$$
h^{\mathrm{T}} \alpha+\mathrm{g}^{\mathrm{T}} \beta+^{\mathrm{T}}+
$$

\section{Analysis of Robust Optimization in Operational Safety Cost of Wind Farms}

\subsection{Robust optimal scheduling of power systems with wind farms}

\subsubsection{Economic dispatch calibration cost model}

The total running cost of the system includes basic running cost and dispatch correction cost, the basic operating cost is the unit generation cost and the abandoned wind deviation cost in the objective function of the robust scheduling model. On the basis of the robust scheduling model, the penalty cost of wind abandoning and load cutting in the simulation scenario is added into the objective function. The unit output and the wind power output in this scenario are calculated according to the calibration cost model, and the economy of the output plan is obtained by analyzing the robust scheduling model. Comparing the simulated wind power output with the wind power allowable output interval obtained by the dispatch model, if the wind power output is within the allowable output interval, wind farms will work as planned. If the wind power output is higher than the allowable output limit, the abandoned wind value can be obtained at the moment according to the allowable upper limit of the output force. Then calculate the corresponding unit adjustment cost and the penalty cost of wind abandoning and load cutting. The objective function of calibration cost model is:

$\operatorname{Min}\{\}$

is the force of the wind farm in the simulated scenario

\subsubsection{Robust optimal scheduling model}

The constraint condition of its power balance is

\subsection{Robust optimal configuration of wind farm energy storage device}

\subsubsection{Robust linear optimization}

Robust linear optimization (RLO) describes the random variables with box constraints, so that the optimization problem is not dependent on its specific distribution, furthermore, the robustness and optimality of the optimal solution are balanced by introducing robustness indicator to adjust the range of random variables, which has a strong practical value in engineering. However, it cannot be 
completely applied to the actual system as the range of uncertain parameter values must be mean value of a symmetrical. Take the general model of minimizing linear programming as an example:

$$
\begin{aligned}
& \operatorname{minf}=c x \\
& \text { s.t. } A x \leq b \\
& 1 \leq x \leq u
\end{aligned}
$$

In the formula, $\mathrm{f}$ is the optimization objective function; $\mathrm{X}$ is the decision variable ( $\mathrm{K}$ dimension column vector), and $\mathrm{kx}$ is the element of $\mathrm{x}$. $\mathrm{U}$ and $\mathrm{l}$ are upper and lower limits of $\mathrm{x}$ respectively. The coefficient matrix $\mathrm{c}$ is the $\mathrm{k}$-dimensional row vector, $\mathrm{b}$ is the $\mathrm{m}$-dimensional column vector, and $\mathrm{A}$ is the m-row k-column matrix. To avoid loss of generality, assume that the uncertain parameters only appear in the elements of the coefficient matrix A. Assume that the element $\mathrm{a}_{\mathrm{mk}}$ of $\mathrm{m}$-row and k-column in A is an uncertain parameter, and the upper limit, lower limit and mean value mk of its value range are known. Record the m-row of uncertain elements $\mathrm{a}_{\mathrm{mk}}$ consisting of $\mathrm{J}_{\mathrm{m}}$, the number of elements in the set is $\mathrm{K}_{\mathrm{m}}$, and it satisfies:

$$
0 \leq \mathrm{K}_{\mathrm{m}} \leq \mathrm{K}
$$

So for the m-row element of matrix A, we can introduce the variable constraint parameter:

$$
\Gamma_{\mathrm{m}}\left(0 \leq \Gamma_{\mathrm{m}} \leq \mathrm{K}_{\mathrm{m}}\right)
$$

To describe the range of variation of each uncertain parameter.

\subsubsection{The influence of wind power randomness on the safe operation of the system}

(1) Minimum cut load model for wind power output randomness

In order to analyze the influence regarding the fluctuation of the output power of multiple wind power plants on the safety of power grid operation, this paper adopts the vector model of minimum tangential load proposed in the literature ${ }^{[1]}$. Based on the linear relationship between the dc power flow and the node injection power, the vector model can be reduced. Then, the calculation model of minimum tangential load, which takes into account the randomness of wind power output, can be described as:

$$
\begin{array}{r}
\min \\
++\leq_{\mathrm{lo}}+, \\
\mathrm{Lo}=1, \cdots, \quad \mathrm{L}
\end{array}
$$

(2) The Conversion of Robust Counterpart

First of all, for the wind farm $\mathrm{j}(1 \leq \mathrm{j} \leq \mathrm{W})$ using the interval to describe the randomness of the output of the wind farm, that is satisfied:

$$
\mathrm{W} \in[\mathrm{W}, \mathrm{W}]
$$

Robust peer model in the change of the constraint in the process of transformation into the same $\Gamma$ constraint parameters, the value range is $0 \leq \Gamma \leq \mathrm{W}$. In order to cover all the possible output of wind farm j, Ujw can take the installed capacity of wind farm and Ljw can take 0 . Jw, as the average power of the wind farm $\mathrm{j}$, can be based on the historical average wind speed, wind power and wind speed curve, this information reflects the wind farm grid region the average wind resources conditions, has statistical significance.

\subsection{Application of robust optimization in power system generation plan}

\subsubsection{Overview of modeling methods for uncertain power generation plan}

Robust optimization does not need to be given the probability distribution of uncertain parameters in advance, but describes the fluctuation of parameters through an uncertain set. As long as the value of the parameter is within the range of uncertain set, the solution of the robust optimization model is feasible. The uncertainty set is a deterministic and bounded set describing the fluctuation range of uncertain parameters. The purpose of robust optimization is to obtain a solution 
that makes any implementation of an uncertain parameter feasible within an uncertain set ${ }^{[2]}$. Compared with the standby criterion method, robust optimization can ensure the reliability of uncertain events; In comparison with stochastic optimization and chance constrained programming, the amount of computation to obtain the optimal solution is greatly reduced, and the demand for the characteristics of uncertain parameters is also significantly reduced. Only its fluctuation range is required without its exact probability distribution.

\subsubsection{Calculation and probability information}

Robust optimization does not require prior knowledge of the probability distribution of uncertain parameters, which is an advantage. However, although the probability distribution cannot be accurately known in practice, there is always some probability information that can be obtained ${ }^{[3]}$. The existing robust optimization model only uses information such as the value range of uncertain parameters, but abandons the rest information, which may lead to a more conservative solution.Or, it directly combines with stochastic optimization methods in order to make use of probability information, and its advantages of fast robust optimization calculation are lost as well. In fact, the approximate probability distribution of uncertain parameters can be obtained by theoretical analysis or mathematical statistics, and the decision-makers also have certain tolerance to the inaccuracy of the approximate distribution ${ }^{[4]}$.

\subsubsection{Risk considerations}

The robust optimization of the uncertain set is a strong requirement: the robust solution is feasible for the realization of uncertain parameters in the uncertain set, and the implementation of uncertain parameters out of the uncertain set is not taken into account ${ }^{[5]}$. This requirement is not totally consistent with reality. On the other hand, there are errors and human intervention in the formulation of the uncertainty set. The true value of the uncertain parameters may fall out of the uncertainty set, which will lead to risks that are difficult to predict or control ${ }^{[6]}$. Therefore, there is a need for an optimization theory that can flexibly adjust the requirements for robustness and quantitatively account for the risks of decision making.

\section{Conclusion}

In short, robust optimization plays a very important role in the safe operation cost of wind farms. Since operational safety is a very important task for wind farms with related to the safety and benefits of power supply, they should be given high priority.

\section{References}

[1] Ji F, Cai XG, Wang CZ. Wind Power System Dispatching Method Based on Weak Robust Optimization. Proceedings of the CSEE 2016; 36:4600-9.

[2] Mei SW, Guo WT, Wang YY, Liu F, Wei W. Game Model and Application Examples of a Class of Robust Optimization Problems in Power System. Proceedings of the CSEE 2013; 33:47-56+20.

[3] Zhang QW, Wang XL, Yang TT, Ren J, Zhang XD. Robust Optimal Scheduling of Power Systems with Wind Farms. Power System Technology 2017; 41:1451-63.

[4] Zhu GY, Lin JK, Luo ZQ, Dai S, Qing L, Liu C. Review on Application of Robust Optimization in Power System Power Generation Planning. Proceedings of the CSEE 2017; 37:5881-92.

[5] Nie HZ, Zhao Y, Ma JB. Consideration of Emergency Demand Side Response and Robust Optimization of Transmission Network Planning When Wind Power Connected to the Grid. Adv Technol Electral Eng Energ 2015; 34:7-11+23.

[6] Han XN, Chen Y, Wen JY. Robust Optimal Configuration of Wind Farm Energy Storage Device. High Voltage Engineering 2015; 41:2217-24. 